<원 저>

\title{
Oral contrast media for computed tomography of canine pancreas
}

\author{
Jihye Choi ${ }^{1}$, Jinhwa Chang ${ }^{2}$, Sunkyoung $\mathrm{Oh}^{2}$, Junghee Yoon ${ }^{2, *}$ \\ ${ }^{1}$ College of Veterinary Medicine, Chonnam National University, Gwangju 500-757, Korea \\ ${ }^{2}$ College of Veterinary Medicine, Seoul National University, Seoul 151-742, Korea
}

(Accepted: March 29, 2011)

\begin{abstract}
Barium suspension, oral iodine contrast medium and water were applied in eight dogs to evaluate (1) distension of gastrointestinal tract, (2) the effect of the oral contrast media on the identification of the pancreas from surrounding organs, and (3) image quality and the presence of artifacts in canine pancreas computed tomography (CT) images. Oral iodine contrast medium, gastrografin, produced significant artifacts that deteriorated the CT images of the pancreas. The use of water did not provide the fullness of the gastrointestinal lumens. Barium suspension was effective for the identification of the pancreas from the surrounding gastrointestinal tract, without significantly increasing image noise. Barium suspension can be used as an optimal contrast medium that will not cause an adverse effect on the pancreatic density and image quality.
\end{abstract}

Keywords : barium, computed tomography, dog, oral contrast media, pancreas

\section{Introduction}

Use of an oral contrast medium for the computed tomography $(\mathrm{CT})$ examination is recommended in most abdominal cases in human patients, and a positive oral contrast medium such as a dilute barium suspension or water-soluble contrast medium containing iodine is conventionally and effectively used $[8,9,11,13]$. Water and less commonly fat density products such as corn oil and milk can be used as negative contrast media $[2,6$, $9,13]$. The essential requirements of oral contrast media should not produce artifacts [12].

The CT examination has been considered as the standard method for pancreatitis $[1,3,5,7]$. However, even in CT images, a contracted and unenhanced duodenum may mimic a pancreatic mass and oral contrast media can be required to examine the pseudotumor effect of the emptying gastrointestinal tract and for the evaluation of the resectability of a pancreatic tumor from the surrounding gastrointestinal tract [12].

We performed this study to investigate the applicability of contrast media to distinguish the pancreas from the adjacent gastrointestinal tract, and to choose an oral contrast medium that produced the least effect on the density of the pancreas and produced minimal artifacts that interfered with the image quality.

\section{Materials and Methods}

\section{Animals}

Eight mature, adult beagle dogs weighing $8 \sim 14 \mathrm{~kg}$ were utilized without distinction of gender. They were clinically healthy based on physical examination, complete blood count and serum biochemistry (normal levels of aspartate aminotransferase, alkaline phosphatase, albumin, total bilirubin, total protein, blood urea nitrogen, creatinine, amylase, lipase, calcium, phosphorus, cholesterol). The dogs were fed commercial food and tap water ad libitum and fasted $24 \mathrm{~h}$ prior to the $\mathrm{CT}$ examination. Dogs were cared for according to The Guide for the Care and Use of Laboratory Animals at the Seoul National University, Korea.

\section{Oral Contrast Media}

All dogs were used three times in turn according to contrast media at one-week interval. Barium suspension (Barium 1.5\%, Easy-CT; TaeJun, Korea), gastrografin (Gastrografin 2\%; Schering, Korea) and water were

\footnotetext{
*Corresponding author

Tel: +82-2-880-1265, Fax: +82-2-880-8662

E-mail: heeyoon@snu.ac.kr
} 
used. All dogs were administered a volume of $50 \mathrm{~mL}$ of an oral contrast agent $30 \mathrm{~min}$ before CT imaging and an additional volume of $20 \mathrm{~mL}$ just prior to the examination through the use of a gastric tube by modified previously described protocol [6].

\section{CT Scanning}

After oral contrast medium administration, general anesthesia was induced with $0.5 \mathrm{mg} / \mathrm{kg}$ diazepam (Merode; Donghwa, Korea) and $6 \mathrm{mg} / \mathrm{kg}$ propofol (Pofol; Dongkook, Korea) intravenously, and maintained with isoflurane (Isoflurane; Rhodia Organique Fine, UK). With the dog in dorsal recumbency, CT images were acquired using a CT-e unit (GE Medical Systems, USA) from the tenth thoracic vertebral region to third lumbar vertebral region. Helical sequences were acquired using $120 \mathrm{kVp}$ and $80 \mathrm{~mA}$ with $2 \mathrm{~mm}$ slice thickness and 1.5 pitch factor in all scans. The field of view ranged between 150 and $220 \mathrm{~mm}$. The dogs were hyperventilated with positive pressure ventilation prior to each scan to produce a period of apnea and avoid motion artifacts.

\section{Image Analysis}

All CT images were evaluated at a window width of 400 and a window level of 40 Hounsfield units (HU). The CT images were analyzed separately at the workstation, each by three different radiologists (Choi $\mathrm{JH}$, Chang JH, and Oh SK). The images were presented unpaired and arbitrarily to the reviewers, who were unaware of any image information other than the window setting. The image findings were recorded on standardized data sheets. Each reviewer scored the luminal distension at four sites - the proximal stomach, distal stomach, duodenum, and the jejunum - using a three point scale (1: poor, 2: adequate, 3 : excellent). The reviewers, using the same scale, evaluated the anatomical conspicuity of the proximal and distal stomach, duodenum, pancreas, liver, portal structures, and the jejunum. An artifact was evaluated with a three point score system (1: barely perceptible, 2: noticeable, 3: interpretation limit). The HU value of the pancreas was measured for quantitative assessment of the effect of oral contrast media on the pancreas. An ellipsoid region of interest was drawn over three regions of the pancreatic body under the field of the gastrointestinal tract containing contrast media per a dog, and recorded as a mean and standard deviation. HU value of the pancreas in each contrast agent was compared with HU values of the pancreas in CT images without oral contrast agent.

\section{Statistics}

Mean values from subjective scores and objective values were calculated per the pancreatic images and reviewer for the use of the oral contrast media. Median score differences and objective value differences among the oral contrast types were calculated per image. Twoway ANOVA tests were performed to evaluate differences in scores among the used contrast media and among the reviewers. The HU values of the pancreas in animals given water and in animals given the barium suspension were compared by one-sample statistics. $P$-values less than 0.05 were considered statistically significant.

\section{Results}

Four of total twenty four CT image sets were excluded from this study due to technical problems such as severe motion artifact and a large amount of luminal gas in gastrointestinal tract. In an axial image, the gastrointestinal loops were hyperattenuated with positive contrast media and hypoattenuated with negative contrast medium (Fig. 1). This change of attenuation made the wall of gastrointestinal tract identifiable from the pancreas. Gastrointestinal distention and fullness were optimal in dogs given gastrografin $(p<0.001)$ (Table 1). There was good interobserver agreement with a $4 / 4$ consensus achieved for 20 images. The anatomical conspicuity of the proximal stomach, distal stomach, and the first two parts of the duodenum, the pancreas, the liver, the portal vein and the jejunum was significantly better with barium suspension than gastrografin and water $(p<0.05)$ (Table 2). There was good interobserver agreement with a $5 / 7$ or $7 / 7$ consensus achieved, respectively. Several broad hypoattenuating streaks superimposed on the pancreas were visible (Fig. 2). The

Table 1. Score for the gastrointestinal distention and fullness assessments according to oral contrast media

\begin{tabular}{lccc}
\hline \hline & Barium & Gastrografin & Water \\
\hline Proximal stomach & 1.94 & $2.30^{*}$ & 2.00 \\
Distal stomach & 1.88 & $2.30^{*}$ & 1.75 \\
Duodenum & 2.13 & $2.75^{*}$ & 1.50 \\
Jejunum & 1.88 & $2.65^{*}$ & 1.38 \\
\hline
\end{tabular}

1: poor, 2: adequate, 3: excellent.

*Significant difference $(p<0.001)$. All data is presented as means. 

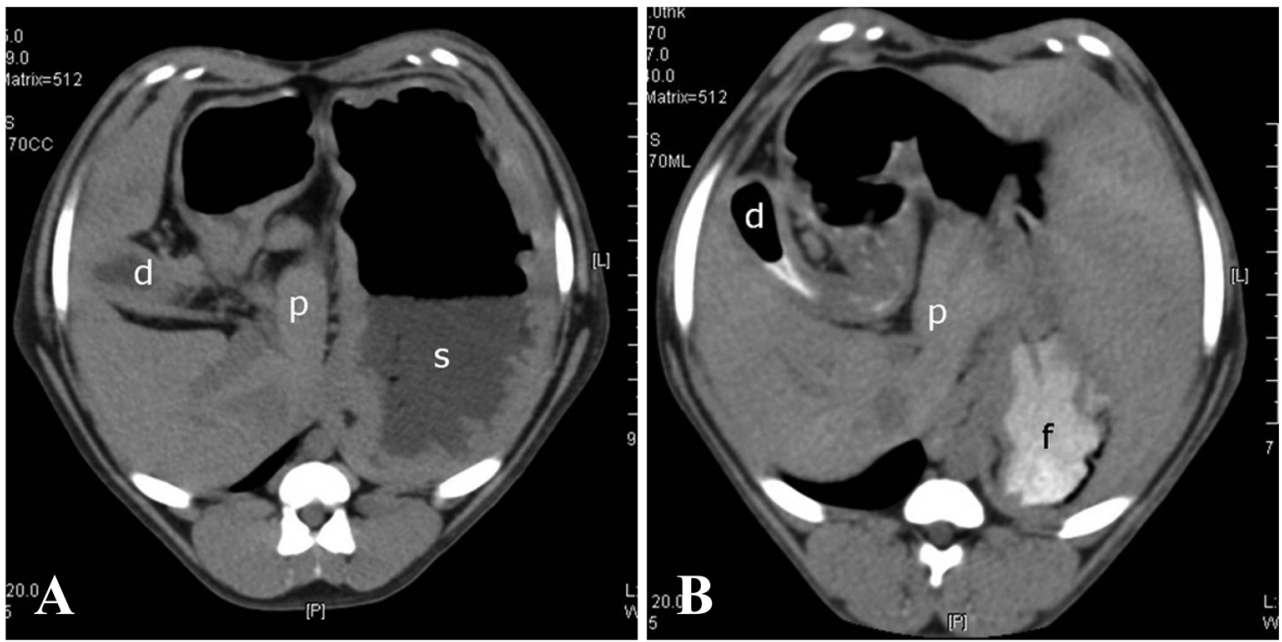

Fig. 1. Axial CT section through the upper abdomen in a normal dog after ingestion of $70 \mathrm{~mL}$ of water (A) and barium (B) as oral contrast medium. Each agent is identified as hyperattenuating and hypoattenuating in gastrointestinal lumen, respectively, and discriminates the pancreas (p) from the stomach (s), gastric fundus (f) and duodenum (d).

Table 2. Score for identification of the pancreas and adjacent organs in oral contrast enhanced CT image

\begin{tabular}{lccc}
\hline \hline & Barium & Gastrografin & Water \\
\hline Proximal stomach & $2.50^{*}$ & 2.30 & 2.19 \\
Distal stomach & $2.56^{*}$ & 2.25 & 2.19 \\
Duodenum & $2.75^{*}$ & 2.20 & 2.06 \\
Pancreas & $1.94^{*}$ & 1.50 & 1.88 \\
Liver & $2.69^{*}$ & 2.20 & 2.44 \\
Portal structures & $2.25^{*}$ & 1.70 & 2.13 \\
Jejunum & $2.13^{*}$ & 2.10 & 1.94 \\
\hline
\end{tabular}

1: barely perceptible, 2 : noticeable, 3 : interpretation limit. *Significant difference $(p<0.05)$. All data is presented as means.

severity of these artifacts varied according to an anatomic location in the same dog. In general, streaks were more pronounced in dogs given gastrografin, and the median artifact score was 2.10, which was significantly higher than score of barium suspension (1.50) and water (1.13) $(p<0.001)$. Water caused no artifact for a CT image as compared with barium $(p<$ 0.05 ). In the quantitative assessment of artifacts, the HU value of the pancreas was $53 \pm 4.83$ with barium suspension and $52 \pm 5.41$ with water, and there was no significant difference from the HU of the pancreas, 52 \pm 4.58 , with no contrast agent. However, the HU values of the pancreas for the dogs given gastrografin had a wide variation, so a significantly consistent $\mathrm{HU}$ value

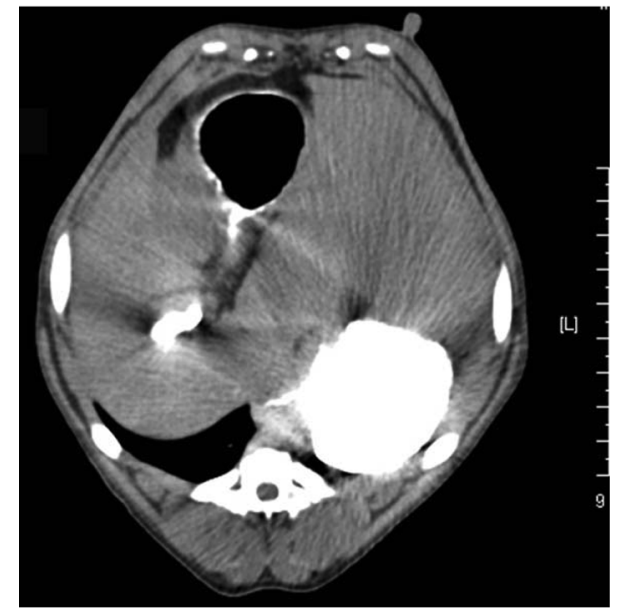

Fig. 2. Axial CT section through the upper abdomen in a normal dog after ingestion of $70 \mathrm{~mL}$ of gastrografin. The streak artifact is observed overall CT image and the pancreas cannot be identified from surrounding structures.

of pancreas could not be measured. The readers agreed on these observations for $75 \sim 83 \%$ of the 20 images.

\section{Discussion}

We applied a modified protocol from a gastrointestinal study in human, in which a volume of $400 \mathrm{~mL}$ of oral contrast agent is ingested from $40 \mathrm{~min}$ to $1 \mathrm{~h}$ before imaging and an additional volume of $200 \mathrm{~mL}$ is ingested 
20 min just prior to the examination [12]. Because this study used oral contrast media for the pancreas CT examination, we reduced volume of contrast media to prevent over-distension and fullness of the stomach and duodenum, which can obscure the pancreas.

The gastrointestinal tract was distended fully enough to identify each other structure and a good coating of the mucous membranes was achieved with the use of the barium suspension and gastrografin. However, rapid passage of water results in inferior distention of the duodenum and difficulties in discrimination from the pancreas. Muscle relaxants such as buscopan can be used to delay the passage of water in the gastrointestinal tract, but even with the use of a muscle relaxant does not provide as good quality images as the use of positive contrast media [12]. Mural visualization with the use of water was inferior to positive contrast media, which have an inherent contrast between mural density and contrast density. Although it is inconvenient but, a contrast enhanced CT scanning with an iodinated intravenous contrast agent can be used concurrently with oral contrast study to compensate a low contrast of water for the gastrointestinal tract through enhancing the mucosal layer [13].

Positive contrast media may increase the local absorbed dose of the X-ray beam in the intestinal mucosa by as much as five times as a result of photoelectric interactions at the contrast agent-mucosa interface, and the streak artifact was exaggerated in dogs given positive contrast media [5]. Gastrografin produced significantly more hypoattenuating linear artifacts than barium suspension, which obscured the depiction of the pancreas on CT images.

It can be presumed that because of the irregular distribution of gastrografin and its high density, a small but highly attenuating agent, that only a part of the slice width, may cause both a partial volume artifact and beam hardening. Gastrografin reduced the HU value of the pancreas, so the HU value of the pancreas could not be used as a relatively objective factor to identify the pathologic lesion in pancreatic parenchyma. This artifact can be an obstacle to evaluate pancreatitis with local complications such as focal fluid collection, a pancreatic pseudocyst and necrosis or a pancreatic mass. To reduce this artifact, a reconstruction algorithm can be used to correct for beam hardening changes [5]. By reducing the slice thickness, fewer attenuation changes are encompassed within the slice volume and a partial volume artifact can be minimized [4]. However, decreasing the slice thickness increases image noise, which is evident as graininess and reformatting requires considerably more slices per a pancreas scan, resulting in longer scan time and increased tube load [10]. CT images could be obtained without a streak artifact with water [9].

This study has limitation that the evaluation of the pancreas $\mathrm{CT}$ images was not performed according to various amounts of contrast media. Fatty agents such as milk or corn oil emulsion were not evaluated in this study, because these agents have a high caloric content and fat, which is not suitable for use in patients with acute pancreatitis $[9,12]$. Milk contains relatively low calories and low fat, but it necessitates the use of an intravenous contrast agent like with water [12].

\section{Conclusion}

The best mural visualization and gastrointestinal distension were achieved with the use of the barium suspension and gastrografin. Gastrografin produced noticeable streak artifacts and affected the pancreatic density, so it cannot be the first choice for the examination of the pancreas. The use of barium suspension for CT of the canine pancreas enhanced diagnostic quality without a significant increase in image noise. Water can considered to be used with a contrast enhanced $\mathrm{CT}$ with intravenous contrast medium, and distention of the bowel with a muscle relaxant.

\section{References}

1. Arvanitakis M, Delhaye M, De Maertelaere V, Bali M, Winant C, Coppens E, Jeanmart J, Zalcman M, Van Gansbeke D, Devière J, Matos C. Computed Tomography and magnetic resonance imaging in the assessment of acute pancreatitis. Gastroenterology 2004, 126, 715-723.

2. Aschoff AJ, Görich J, Sokiranski R, Rieber A, Brambs HJ, Krämer SC. Pancreas: Does hyoscyamine butylbromide increase the diagnostic value of helical CT? Radiology 1999, 210, 861-864.

3. Balthazar EJ, Robinson DL, Megibow AJ, Ranson JH. Acute pancreatitis: Value of CT in establishing prognosis. Radiology 1990, 174, 331-336.

4. Glover GH, Pelc NJ. Nonlinear partial volume artifacts in X-ray computed tomography. Med Phys 1980, 7, 
238-248.

5. Hathcock JT, Stickle RL. Principles and concepts of computed tomography. Vet Clin North Am Small Anim Pract 1993, 23, 399-415.

6. Hofer M. CT Teaching Manual. 1st ed. pp. 10-14, Thieme, New York, 2000.

7. Imbriaco M, Megibow AJ, Camera L, Pace L, Mainenti PP, Romano M, Selva G, Salvatore M. Dual-phase versus single-phase helical CT to detect and assess resectability of pancreatic carcinoma. Am J Roentgenol 2002, 178, 1473-1479.

8. Owens JM, Biery DN. Radiographic Interpretation for the Small Animal Clinician. 2nd ed. pp. 1-8, Williams \& Wilkins, Pennsylvania, 1998

9. Ramsay DW, Markham DH, Morgan B, Rodgers PM, Liddicoat AJ. The use of dilute Calogen as a fat density oral contrast medium in upper abdominal computed tomography, compared with the use of water and positive oral contrast media. Clin Radiol 2001, 56, 670-673.

10. Silverman PM. Helical (Spiral) Computed Tomography. a Practical Approach to Clinical Protocols. 1st ed. pp. 1-10, Williams \& Wilkins, Philadelphia, 1998.

11. Stickle RL, Hathcock JT. Interpretation of computed tomographic images. Vet Clin North Am Small Anim Pract 1993, 23, 417-435.

12. Thompson SE, Raptopoulos V, Sheiman RL, McNicholas MM, Prassopoulos P. Abdominal helical CT: milk as a low-attenuation oral contrast agent. Radiology 1999, 211, 870-875.

13. Winter TC, Ager JD, Nghiem HV, Hill RS, Harrison SD, Freeny PC. Upper gastrointestinal tract and abdomen: water as an orally administered contrast agent for helical CT. Radiology 1996, 201, 365-370. 